\title{
Gastos com salários e desempenho em matemática: uma análise baseada nas escolas municipais de $\mathrm{SBC}^{1}$
}

\author{
Fernando Vizotto Galvão²
}

\section{Resumo}

Este trabalho explora a relação entre gastos com educação e desempenho escolar, a partir de informações de gastos no nível das escolas. A análise da relação entre gastos e desempenho tem se baseado em estimativas que utilizam dados agregados de gastos com educação. Neste artigo, indicamos a importância da informação de gastos no nível da escola para que se possa avançar no sentido de compreender não somente se gastos com educação estão ou não associados ao desempenho, mas como diferentes tipos de gastos estão relacionados de diferentes formas ao desempenho dos alunos. Com base na revisão da literatura pertinente, elaboramos uma proposta de categorias de gastos com pessoal para as escolas da rede municipal de São Bernardo do Campo, sendo as categorias de maior agregação as que dizem respeito ao que consideramos gastos diretamente relacionados ao ensino e gastos indiretamente relacionados ao ensino. A partir das categorias delimitadas, baseadas nas informações de gastos por escola, foram exploradas as relações entre gastos por aluno e desempenho em matemática na Prova Brasil de 2013, por meio de análise de regressão. As estimativas revelaram que a relação entre gastos e desempenho não é homogênea entre as diferentes categorias de gasto e que a especificação dos modelos melhora na medida em que os gastos são desagregados. Dentre os resultados obtidos, destacamos aquele que indica uma relação positiva e significante entre os gastos com coordenação pedagógica e o desempenho em matemática.

\section{Palavras-chave}

Gastos com educação - Desempenho escolar - Gastos no nível da escola - Recursos escolares.

1- Texto adaptado da dissertação apresentada ao Programa de Pós-Graduação da Faculdade de Educação da Universidade de São Paulo para a obtenção do título de Mestre em Educação, desenvolvida sob orientação do Prof. Dr. Romualdo Luiz Portela de Oliveira.

2- Universidade de São Paulo, São Paulo, SP, Brasil. Contato: fernando.galvao@usp.br. 


\section{Personnel expenses and performance in mathematics: an analysis based on the municipal schools of $\mathrm{SBC}$}

\section{Abstract}

This paper aims to explore the relationship between the money spent on education and school performance, based on the expenditure information on the school-level. The debate on the relationship between expense and performance has been mostly based on estimates using the aggregated data of municipal spending on education. In this study, we show the relevance of the information on spending at the school level. With this, we intend not only to understand if education expenses are or are not associated with performance, but also to observe how different types of costs are related in different ways to the performance of students. Based on literature review, we have proposed categories of personnel expenses for the municipal schools of São Bernardo do Campo. Categories of higher aggregation involve what we consider to be expenses directly related to teaching and expenses indirectly related to teaching. From the specific categories, which are based on information on spending per school, we explore the relationship between expenses per student and their performance in the Mathematics exam in Prova Brasil 2013, using regression analysis. The estimates have shown that the relationship between spending and performance is not homogeneous amidst the different categories of expenditure, and that specification of models improves as expenditures are disaggregated. Among the results, we highlight the indication of a positive and significant relation between spending on Pedagogical Coordination and performance on the mathematics exam.

\section{Keywords}

Spending on education - School performance - Expenditure at the school level - School resources.

\section{Introdução}

Desde a publicação dos resultados da pesquisa conduzida pelo sociólogo James S. Coleman, em 1966, que apontou a pouca relevância dos recursos escolares para explicar o desempenho dos alunos em testes, instaurou-se o debate sobre a existência ou não de uma relação entre gastos com educação e desempenho. Nas décadas que se seguiram, a conclusão de que os recursos não importam tem sido recorrente, assim como sua contestação (VIGNOLES et al., 2000). Em geral, não se nega que certa quantidade de recursos é necessária para que as atividades das escolas sejam viabilizadas, mas a análise

3- This text was adapted from the thesis presented to the School of Education of the University of São Paulo as part of the requirements to obtain the Master's Degree in Education having Prof. Romualdo Luiz Portela de Oliveira as my supervisor. 
dos gastos com educação, feita quase sempre a partir de dados agregados de redes de ensino e não de gastos de unidades escolares, muitas vezes reforça a conclusão inicial do estudo de Coleman, de que não há uma relação relevante entre gastos e o desempenho dos alunos (HANUSHEK, 1986; 1997; 2003; AMARAL; MENEZES-FILHO, 2008; AMARAL, 2011; MONTEIRO, 2015).

Diante das conclusões sobre o tema e da ausência de consenso em torno delas (HANUSHEK, 1989; HEDGES; LAINE; GREENWALD, 1994), alguns autores têm indicado a necessidade do deslocamento do debate de se os recursos importam para como os recursos importam (PICUS, 1995; ODDEN et al., 2003). Isto é, seria necessário investigar não somente a relação entre gastos agregados com educação e desempenho médio de alunos de uma rede de ensino, mas avançar no entendimento de como diferentes tipos de gastos, associados a diferentes atividades escolares, estão relacionados de diferentes formas ao desempenho dos alunos. Oliveira (2006) sugere abordagem similar, apontando a necessidade, no Brasil, de pesquisas que procurem valorar os efeitos de diferentes opções de alocação dos recursos educacionais sobre determinados resultados escolares, como aprendizagem ou permanência dos alunos nas escolas.

Para a realização deste tipo de análise, são apontados como necessários dados sobre gastos no nível das escolas, assim como informações que apontem mais para os usos feitos dos recursos do que para categorias econômicas ou contábeis das despesas efetuadas (PICUS; PETERNICK, 1998; KIMBALL, 2009, p. 48). Dito de outra forma, para entender quais atividades escolares os recursos alocados às escolas viabilizam e como podem estar associados à aprendizagem dos alunos, é necessário que a informação de gastos com educação seja obtida a partir das unidades de ensino.

$\mathrm{Na}$ tentativa de avançar em relação ao entendimento das relações entre gastos com educação e o desempenho dos alunos, exploramos as associações entre essas variáveis a partir de dados de gastos e desempenho no nível das escolas, com base em informações fornecidas pela Secretaria da Municipal de Educação da cidade de São Bernardo do Campo - SBC. As páginas que se seguem tratam de uma breve análise da literatura sobre a informação de gastos no nível das escolas, da abordagem metodológica seguida e dos resultados obtidos, no que diz respeito às relações entre gastos e desempenho. Por fim, são indicadas as principais conclusões, as limitações da pesquisa e algumas possibilidades de caminhos a serem seguidos em pesquisas futuras.

\section{Os gastos das escolas}

No Brasil, não são muitos os trabalhos que se dedicam à análise dos gastos das escolas e os que o fazem, em geral, não têm a intenção de estabelecer uma relação entre gastos e o desempenho dos alunos. Estas pesquisas, na maior parte dos casos, estão interessadas em conhecer os gastos de escolas de diferentes tipos - no que diz respeito a tamanho (número de alunos), localização (rural ou urbana), região do país, etapa e modalidade do ensino básico, ou a fatores indicativos da qualidade do ensino oferecido (como taxa de docentes com formação superior ou número médio de horas-aula diárias, por exemplo) - para tentar encontrar algum padrão de gasto, ou mais precisamente de 
custo, que possa servir de referência para propostas ou decisões sobre o financiamento da educação básica (ALVES, 2012, p. 131; GALVÃ0, 2016, p. 14-27). Tais trabalhos dedicamse a mensurar o que tem sido chamado de custo-aluno (PARO, 1981; XAVIER; MARQUES, 1988; VERHINE, 2006), o que de fato fazem, com maior ou menor precisão, a depender da informação disponível (ALVES, 2012, p. 131-132; GALVÃO, 2016, p. 31-32).

Diferente das pesquisas que tratam dos gastos ou despesas públicas agregadas (por município, estado ou país) por aluno, estes trabalhos procuram delimitar as despesas que podem ser atribuídas a determinada escola, segundo a etapa de ensino, em determinado ano. Para isso, são mensuradas: 1) as despesas correntes de cada unidade de ensino, como as relativas à água, luz, alimentação dos alunos e, sobretudo, as que dizem respeito aos salários, que correspondem à maior parcela dos gastos das escolas (VERHINE, 1998, p. 112); e 2) a depreciação dos ativos disponíveis nas escolas, o que indica a dimensão da parcela da despesa de capital a ser atribuída a cada escola no ano considerado. 0 direcionamento das despesas a determinada etapa ou modalidade de ensino, quando necessário, é feito por meio de rateios, que se baseiam no número de alunos (GALVÃo, 2016, p. 31-32).

Vale notar que, tendo em vista o estabelecimento de uma relação do tipo insumoproduto, como é o caso das pesquisas de viés econômico que tratam da relação entre gastos e desempenho dos alunos, a informação de custo por aluno pode ser mais precisa do que a informação de despesa pública com educação, principalmente porque esta última atribui às escolas o total de despesas com compras de materiais e equipamentos efetuados em determinado período, gastos estes que não terão efeitos proporcionais sobre o desempenho dos alunos no mesmo período. Além disso, a despesa pública realizada em determinado ano deixa de levar em conta os gastos passados com compra de materiais e equipamentos que já estão disponíveis nas escolas, os quais de fato viabilizaram as atividades escolares no período tomado como referência. Em resumo, pode-se dizer que a consideração da despesa pública com a compra de materiais e equipamentos em determinado período e a desconsideração do custo relativo aos materiais e equipamentos presentes nas escolas torna passível de ressalvas as estimativas que utilizam dados de despesas públicas com educação para explorar a relação entre tais despesas e o desempenho dos alunos.

De toda forma, como já indicado, as pesquisas de custo-aluno realizadas no Brasil, apesar de tratarem em detalhe da informação de custos no nível das escolas, não foram delineadas com o intuito de estabelecer relações entre gastos e desempenho. Por sua vez, as pesquisas que se dedicam à mensuração deste tipo de relação fazem uso de dados de gastos com Ensino Fundamental agregados por município e da informação de desempenho médio municipal dos alunos em testes de larga escala, como a Prova Brasil (AMARAL; MENEZES-FILHO, 2008; AMARAL, 2011; MONTEIRO, 2015). Tendo isto em vista, para avançar em relação a abordagens que tratam de gastos no nível das escolas e desempenho dos alunos, é necessário recorrer a outros contextos.

Archibald (2006) empreende pesquisa que, a partir de informações de gastos de unidades de ensino, mensura a relação entre gastos com educação e desempenho dos alunos. Utilizando dados sobre os gastos de 53 escolas de ensino fundamental de um distrito da cidade norte-americana de Reno, a autora estima, por meio de modelos hierárquicos lineares de três níveis (aluno, sala de aula e escola), a relação entre diferentes 
tipos de gastos efetuados pelas escolas e o desempenho dos alunos em testes de leitura e matemática. As estimativas levam em consideração os gastos com ensino ${ }^{4}$, suporte ao ensino $^{5}$, liderança/chefia ${ }^{6}$ e operações e manutenção ${ }^{7}$, além de incluírem variáveis de contexto dos alunos e das escolas. Os resultados obtidos apontam para efeitos positivos e significantes do gasto por aluno com ensino e com suporte ao ensino, assim como do gasto total por aluno, sobre o desempenho dos estudantes em leitura.

Como é possível notar, a desagregação dos gastos em diferentes categorias permite uma maior compreensão acerca do que está ou não associado ao desempenho, para além do gasto total. A respeito da utilização de dados no nível das escolas, a autora aponta que:

0 fato deste modelo utilizar dados de gasto por aluno no nível da escola, em uma estrutura de níveis, indica que esta estimativa de efeito dos recursos sobre o desempenho é melhor do que a de muitos estudos anteriores, que usaram dados agregados no nível do distrito. (ARCHIBALD, 2006, p. 34, tradução nossa).

Em relação às categorias de gastos utilizadas, os resultados obtidos por Archibald (2006) confirmam uma tendência sugerida em outros estudos: os gastos com educação tendem a ser mais relevantes em relação ao desempenho quando viabilizam atividades escolares mais diretamente relacionadas ao ensino (FERGUSON, 1991, p. 485; CONLEY; PICUS, 2003; PICUS et al., 2008). Isso não significa que gastos não diretamente relacionados ao ensino não sejam relevantes, mesmo porque diversas atividades diretamente relacionadas ao ensino são viabilizadas por eles. Mas indica que um dos resultados possíveis das atividades desenvolvidas nas escolas - o desempenho satisfatório em testes, que pode ser entendido como uma proxy para a aprendizagem dos alunos (ver OLIVEIRA et al., 2013, p. 21) - tende a estar positivamente associado aos gastos que viabilizam atividades mais diretamente ligadas ao ensino dos alunos. Dito de outra forma, é sempre válido lembrar que as atividades e os resultados escolares não se resumem aos aspectos cognitivos dos alunos e que os gastos necessários para viabilizar tantas outras funções das escolas podem ser tão importantes quanto os destinados às atividades de ensino de português e matemática (BENEVIDES, 1996; CARVALHO, 2015).

0 tipo de análise dos gastos no nível da escola, conforme empreendido por Archibald (2006), segundo agregações que tendem a indicar a associação entre o gasto e a atividade escolar viabilizada, tem sido apontado como essencial para o entendimento de como diferentes opções de financiamento ou de alocação dos recursos educacionais podem afetar os resultados escolares dos alunos (PICUS; PETERNICK, 1998, p. 109; ODDEN et

\footnotetext{
4- "Isso inclui financiamento para professores, professores substitutos, profissionais de apoio ao ensino, tecnologia da informação para uso dos alunos, materiais utilizados para o ensino, viagens e suprimentos" (p. 40, tradução nossa).

5 - "Isso inclui financiamento para orientação e aconselhamento, bibliotecas e mídia, atividades extracurriculares, serviços de saúde para os alunos, desenvolvimento do currículo, desenvolvimento profissional [da equipe escolar], licenças, gestão de programas [escolares], terapeutas, psicólogos, avaliadores e assistentes sociais" (p. 40, tradução nossa).

6 - "Isso inclui financiamento para diretores, vice-diretores, suporte administrativo, deputados, administradores sêniores, pesquisadores, avaliadores de programas, superintendentes, representantes do conselho escolar e equipe jurídica" (p. 40, tradução nossa).

7- "Isso inclui financiamento para transporte, alimentação, segurança, conservação do prédio escolar, utilidades (água, luz, telefone etc.), manutenção, processamento de dados e despesas administrativas gerais" (p. 40, tradução nossa).
} 
al., 2003). Isto é, saber com o que são gastos os recursos destinados à educação é menos relevante do que saber para que são gastos tais recursos, quando se deseja entender como gastos com educação e desempenho escolar se relacionam.

Dito ainda de outra forma, se escolas ou sistemas de ensino desejam saber como a alocação dos recursos disponíveis pode influenciar a melhoria da aprendizagem, o conhecimento dos gastos com atividades de direção ou coordenação escolar, ou ainda com aulas de matemática ou atividades de reforço, tem mais a dizer do que o conhecimento dos gastos com pessoal ou materiais de consumo. A informação do gasto associado às atividades ou funções escolares, contudo, não faz sentido em agregações superiores à escola, pois estas atividades e suas consequências, em termos de aprendizagem, se dão justamente nas escolas. Ou seja, a informação da despesa agregada com educação (por distrito, município, estado ou país) tem muito pouco a dizer a respeito do que foi feito, nas escolas, com os recursos viabilizados via financiamento, tornando frágeis as estimativas que procuram estabelecer relações entre gastos e desempenho dos alunos com base em dados de gastos agregados com educação (HANUSHEK; RIVKIN; TAYLOR, 1996; PICUS et al., 2008, p. 6).

Procurando avançar no entendimento das relações entre gastos e desempenho no nível das escolas, alguns estudos têm se dedicado a acompanhar mudanças feitas na alocação dos recursos de unidades de ensino que passaram por reformulações de seu financiamento, mudanças estas que tinham por objetivo a disponibilização de recursos que fosse compativel com melhorias desejadas no desempenho dos alunos (PICUS; PETERNICK, 1998; CONLEY; PICUS, 2003; ODDEN et al., 2003; PICUS et al., 2008). Empreendidas no contexto das políticas de adequacy of funding ${ }^{8}$, que geralmente procuram determinar o financiamento adequado às necessidades das escolas no contexto norte-americano (inclusive tendo em vista os resultados escolares a serem alcançados), estas pesquisas dedicam-se a acompanhar os gastos das escolas durante certo período e verificar em que medida as variações no padrão de gastos influenciam os resultados escolares ou são suficientes para sustentar os resultados pretendidos. Para tanto, os gastos das escolas são classificados de acordo com determinadas atividades ou funções a serem desempenhadas pelas escolas e, paralelamente, são estabelecidos indicadores que possam captar os usos feitos dos recursos.

Em uma destas pesquisas, Odden et al. (2003), com base em pares de escolas que atendem alunos de diferentes condições sociais e que passaram por reformulação de suas estratégias de alocação dos recursos, propõem um conjunto de indicadores e de categorias de gastos escolares (anexos A e B) que apontam ser apropriados para o acompanhamento da estratégia de alocação dos recursos, tendo em vista a análise das relações entre os gastos das escolas e a aprendizagem dos alunos. Em linhas gerais, pode-se dizer que os autores identificam grupos de atividades ou funções escolares que podem afetar, de forma mais direta ou mais indireta, a aprendizagem dos alunos, e indicam que o gasto deve ser associado a essas atividades, cujos maiores agrupamentos correspondem aos gastos relacionados ao ensino e não relacionados ao ensino.

8- No início da década de 1990, cortes de diversos estados norte-americanos passaram a indicar a necessidade de adequações dos sistemas estaduais de ensino. Essas adequações geralmente incluem mudanças no financiamento das escolas e consideram os resultados que os sistemas de ensino devem garantir (KIMBAL, 2009, p. 39-44). 
A proposta de categorias de gastos e de indicadores feita por Odden et al. (2003) foi utilizada por Picus et al. (2008) para avaliar se 334 escolas (de um total de 362) do estado norte-americano de Wyoming seguiram determinadas estratégias de alocação de recursos, estratégias estas que deveriam suportar uma melhoria do desempenho dos alunos. A análise dos gastos das escolas indicou que houve certo desvio em relação ao que se pretendia. A estratégia de privilegiar gastos com a contratação de tutores, com base na quantidade de alunos com dificuldade de aprendizagem, em detrimento da contratação de professores auxiliares, por exemplo, não foi seguida. Também não foi seguida a diretriz de ampliação da contratação de professores das matérias consideradas centrais no currículo. Em conclusão, os autores apontaram que, em alguns casos, “[...] mais recursos foram alocados para áreas não relacionadas às atividades de ensino do que o recomendado pelo modelo" (PICUS et al., 2008, p. 27, tradução nossa), o que pode ter implicações negativas em relação à trajetória esperada para o desempenho dos alunos.

Ainda que os autores não tenham se dedicado a investigar as relações entre os gastos das escolas e o desempenho dos alunos, eles fazem uso de categorias de gastos com educação que são úteis para associar recursos destinados às escolas, atividades viabilizadas por estes recursos e resultados a serem proporcionados pelas opções feitas na alocação dos recursos. Tendo isto em vista, consideramos que estas categorias podem ser úteis como referência para análises dedicadas a explorar a relação entre gastos das escolas, segundo as atividades ou funções escolares existentes, e desempenho.

Diante do exposto e considerando a importância de avançar no entendimento acerca da relação entre gastos com educação e desempenho, tomamos como referência as abordagens de tratamento dos gastos escolares no contexto norte-americano, especialmente a proposta por Odden et al. (2003), para explorar a relação entre gastos e desempenho dos alunos, no nível da escola, na rede municipal da cidade de São Bernardo do Campo. Para isso, foram considerados como medida de gastos os salários de cada funcionário, segundo a função exercida, em cada escola da rede, no ano de 2013. A próxima seção indica os passos seguidos para a construção da informação de gasto e a metodologia utilizada para a análise da relação entre gastos e desempenho.

\section{Aspectos metodológicos}

Antes da descrição das opções feitas em relação ao tratamento e análise dos dados, convém um breve comentário sobre a medida escolhida para representar a aprendizagem dos alunos. 0 processo por meio do qual o desempenho em testes de larga escala, como a Prova Brasil, passa a ocupar determinado espaço no debate educacional, é permeado por disputas e está baseado em diferentes representações de educação e de resultados possiveis da educação escolar (OLIVEIRA; ARAUJO, 2005; BONAMINO; SOUSA, 2012; BAUER; ALAVARSE; OLIVEIRA, 2015). Ainda que a problematização deste processo seja importante, não é objetivo deste artigo fazer este tipo de análise. Desta forma, mesmo que tanto o processo de construção do lugar de destaque assumido pelos testes em larga escala quanto as consequência dos usos feitos deste tipo de medida mereçam exame mais detido, entendemos que a informação produzida por tais testes viabiliza análises que podem ser 
úteis para a elaboração de diagnósticos que contribuam para a melhoria do ensino e da aprendizagem, inclusive levando em consideração aspectos relacionados a desigualdades educacionais (OLIVEIRA et al., 2013). Tendo isso em vista, optamos por utilizar a Prova Brasil de 2013 como uma das fontes de informação para a elaboração do presente estudo, cientes de que a análise dos dados deve ser feita sempre com parcimônia. Feita esta breve observação, passamos à descrição da abordagem seguida no tratamento e análise dos dados.

A rede municipal de educação de São Bernardo do Campo contava com 175 escolas de educação infantil e de ensino fundamental em 2013, ano tomado como referência para o levantamento dos gastos. Destas escolas, aproximadamente 70 ofereciam ensino fundamental, o que correspondia a um total de 40.755 alunos matriculados. Para esta pesquisa foram selecionadas as escolas em que mais de 75\% dos alunos participaram da Prova Brasil de 2013 (69 escolas) e, dentre estas, as que ofereciam exclusivamente os anos iniciais do ensino fundamental (61 escolas) e que tinham informações de gastos por escola disponíveis, o que resultou em um total de 55 escolas com dados para análise.

Em relação aos gastos considerados na pesquisa, foi possível somente a coleta de dados relativos às despesas com pagamentos de salários dos profissionais que atuavam nas escolas. Isto é, não foram considerados, por exemplo, as despesas com alimentação, água, luz, materiais utilizados para o ensino e depreciação de móveis e equipamentos existentes em cada unidade de ensino. Por um lado, essa restrição se deu devido ao alto custo de se produzir parte destas informações para cada escola, tendo em vista que os responsáveis pela produção dos dados teriam de realizar rotinas de trabalho adicionais para que estas informações fossem sistematizadas. Por outro lado, parte desses gastos só poderia ser obtida a partir do levantamento dos dados diretamente nas escolas, o que não foi possível, dada a quantidade de escolas a serem visitadas e o número restrito de pessoas para a realização desta tarefa. Contudo, tendo em vista os estudos sobre custo por aluno produzidos no Brasil, que indicam que a despesa com pagamento de salários representa entre 65\% e 85\% do total das despesas das escolas, pode-se dizer que a maior parte dos gastos das escolas foi considerada na pesquisa (LEVY; CAMPINO; NUNES, 1970, p. 151; PAR0, 1981, p. 72; VERHINE, 2006, p. 53).

A despesa com salários, por funcionário, segundo a função exercida, para cada escola, foi obtida para um mês sem pagamentos adicionais (férias e décimo terceiro salário) e, em seguida, anualizada. Com o propósito de tornar as escolas comparáveis, a despesa com salários de funcionários responsáveis pela limpeza foi excluída das escolas que indicavam esse gasto, pois em parte das escolas esse serviço era realizado por funcionários terceirizados. Em relação a outros funcionários terceirizados, não houve menção a algum outro grupo de profissionais que atuasse de forma terceirizada em algumas escolas e, em outras, como servidores diretamente vinculados à rede municipal de ensino, o que minimizou a possibilidade de distorções na comparação dos gastos entre escolas. Ainda tendo em vista os funcionários terceirizados, não foi possível a delimitação dos gastos com profissionais que porventura estivessem atuando nas escolas sob esta forma de contratação (na cozinha ou na segurança, por exemplo), não sendo possível inferir a magnitude dos gastos com pagamento de salários desses funcionários. 
Em relação aos gastos com Educação de Jovens e Adultos - EJA, a opção foi excluir os alunos matriculados nessa modalidade do total de matrículas e excluir os gastos de salários de professores dedicados ao ensino de jovens e adultos, o que foi feito com base na identificação da função exercida por cada professor. A exclusão da parcela de salários dos demais funcionários das escolas foi feita de acordo com a proporção de alunos matriculados em EJA em relação ao total.

Os gastos anualizados foram agregados por escola, segundo categorias associadas a determinadas atividades ou funções escolares (Quadro 1). A relação entre a função exercida por cada funcionário, em cada escola, e a respectiva categoria de gasto consta no anexo C. Tomando como referência a classificação proposta por Odden et al. (2013) e considerando o contexto da rede de ensino em questão, os gastos foram agrupados em diretamente relacionados ao ensino e indiretamente relacionados ao ensino. Como não foram considerados gastos com água, luz, transporte escolar etc., os gastos indiretamente relacionados ao ensino coincidem com os gastos com gestão escolar.

Quadro 1- Categorias de gasto consideradas - Rede Municipal de SBC

Gastos diretamente relacionados ao ensino

Desenvolvimento do currículo básico

Professores

Assistentes de classe

Estagiários

Desenvolvimento de atividades de ensino - Ed. Especial

Professores Ed. Especial

Estagiários Ed. Especial

Espaço para pesquisa, leitura e ensino (biblioteca)

Orientação e suporte às atividades de ensino e aprendizagem

Coordenação pedagógica

Suporte aos alunos e professores (monitoria)

Gastos indiretamente relacionados ao ensino

Gestão escolar

Direção da escola

Apoio operacional e administrativo

Fonte: Elaborada, pelo autor, com base em informações fornecidas pela Secretaria de Educação de SBC.

A análise de regressão foi a técnica utilizada para estimar a relação entre gastos por aluno e o desempenho em matemática na Prova Brasil de 2013. A opção por considerar o desempenho em matemática deve-se ao fato dessa matéria ser tipicamente escolar, sendo seu conteúdo desenvolvido essencialmente na escola. No caso de língua portuguesa, ainda que a escola exerça um papel importante, pode-se entender que a vivência familiar, dentre outras, pode influenciar a aprendizagem, o que pode ter consequências em termos 
de resultados em testes como a Prova Brasil (CHUDGAR; LUSCHEI, 2009). Dessa forma, considerando que pode ser mais difícil isolar os fatores escolares associados ao desempenho dos alunos em língua portuguesa, relativamente ao desempenho em matemática, optamos por utilizar o desempenho em matemática como variável dependente.

Como variável de controle, foi utilizado o Indicador de Nível Socioeconômico INSE $^{9}$ das escolas, conforme divulgado pelo Instituto Nacional de Estudos e Pesquisas Educacionais Anísio Teixeira - INEP (INEP, 2014). Controles adicionais foram incluídos em um dos modelos ${ }^{10}$, mas o número de variáveis consideradas foi restrito, devido ao número de observações (HAIR et al., 2009, p. 168). De toda forma, tendo em vista que o nível socioeconômico dos alunos é apontado como o principal fator explicativo das notas em exames como a Prova Brasil (ALVES; FRANCO, 2008, p. 491), a variável de controle geralmente apontada como mais relevante foi contemplada.

Os modelos estimados tiveram como base a seguinte equação:

$$
\text { Nota em matemática }{ }_{i}=\alpha+\beta \text { Gasto }_{i}+\gamma(\text { INSE })_{i}+\text { demais controles }+\varepsilon_{i}
$$

em que a variável dependente é a nota em matemática na Prova Brasil, Gasto representa o conjunto de variáveis relativas ao gasto por aluno, segundo as categorias propostas, INSE é o Indicador de nível socioeconômico médio de cada escola, e demais controles se refere a variáveis relativas à experiência do diretor ${ }^{11}$ e ao tempo gasto com a manutenção da disciplina em sala de aula ${ }^{12}$.

\section{Resultados}

Os dados agregados, segundo os parâmetros descritos e de acordo com a classificação proposta, foram submetidos à Secretaria de Municipal de Educação de São Bernardo do Campo, que cedeu os dados para a realização da pesquisa. Após o esclarecimento de dúvidas e debate quanto às atividades exercidas nas escolas pelos funcionários, foi possível chegar ao valor do gasto por aluno, de acordo com a classificação proposta, para cada escola considerada.

A Tabela 1 indica os menores e maiores valores do gasto por aluno, expressos em reais de 2013, além da média e do desvio-padrão. Contrariando o que se poderia esperar, o gasto por aluno apresenta variação significativa entre as escolas da rede. Isto é, tendo em vista que a alocação dos funcionários das escolas é feita de forma centralizada, seria

9- 0 Indicador de Nível Socioeconômico das Escolas - INSE é uma síntese de medidas relativas à condição social dos alunos, como, por exemplo, escolaridade dos pais e disponibilidade de determinados bens em suas casas. 0 indicador é expresso em uma escala contínua, mas também em sete níveis, relativos a sete grupos de nível socioeconômico: Muito baixo, Baixo, Médio Baixo, Médio, Médio alto, Alto e Muito alto.

10- Os controles adicionais foram selecionados com base na comparação dos modelos ( $R^{2}$ e teste $F$ ).

11- A variável relativa à experiência do diretor diz respeito aos anos de experiência que o diretor da escola tem nesta função (item 16 do questionário do diretor da Prova Brasil de 2013). A variável foi dividida em 3 categorias: diretores com até 2 anos de experiência; diretores com 3 a 10 anos de experiência; e diretores com 11 anos ou mais de experiência.

12- A variável relativa ao tempo gasto com manutenção da disciplina em sala de aula diz respeito à proporção de professores, por escola, que indicaram passar menos de 10\% do tempo em sala de aula mantendo a ordem/disciplina (item 103 do questionário do professor da Prova Brasil de 2013). 
possível supor que o gasto com salários por aluno fosse similar entre as escolas. As razões para as diferenças podem ser muitas: atendimento a diferentes necessidades de recursos, em linha com diferentes necessidades de acesso e aprendizagem; profissionais mais experientes e com maiores salários atuando em determinadas escolas - motivados pela localização, pelo perfil dos alunos, pelas condições de trabalho etc. -, supondo que possam escolher a escola em que vão trabalhar; tamanhos diferentes de escolas e de turmas, a depender da infraestrutura disponível em cada local etc.

Tabela 1- Gasto por escola segundo as categorias de gasto consideradas - rede municipal de SBC (2013)

\begin{tabular}{l|c:cc:c}
\hline & Min. & Max. & Média & Desvio-padrão \\
\hline Gasto por Aluno - Total & $1.516,85$ & $3.277,67$ & $2.171,59$ & 352,76 \\
\hline Gasto diretamente relacionado ao ensino & $1.316,26$ & $2.773,16$ & $1.844,57$ & 277,40 \\
\hline Desenvolvimento do currículo básico & $1.032,27$ & $1.953,59$ & $1.371,82$ & 178,44 \\
\hline Desenvolvimento de atividades de ensino - Ed. Especial & 0,00 & 594,52 & 127,58 & 93,30 \\
\hline Espaço para pesquisa, leitura e ensino (biblioteca/sala de leitura) & 0,00 & 172,17 & 44,52 & 29,85 \\
\hline Orientação e suporte às atividades de ensino e aprendizagem & 174,59 & 518,72 & 300,65 & 83,32 \\
\hline Gestão escolar & 187,24 & 816,25 & 327,02 & 115,33 \\
\hline
\end{tabular}

Fonte: Elaborado, pelo autor, com base em informações fornecidas pela Secretaria de Educação de SBC e INEP.

Em relação à distribuição dos gastos entre as diferentes categorias (Gráfıco 1), consideradas todas as escolas, pode-se notar que a maior proporção dos gastos ocorre em Desenvolvimento do currículo básico, que concentra a remuneração dos professores. Nota-se também a similaridade entre os percentuais de gastos com Orientação e suporte às atividades de ensino e aprendizagem e Gestão escolar.

Gráfico 1 - Distribuição do gasto por categoria - rede municipal de SBC
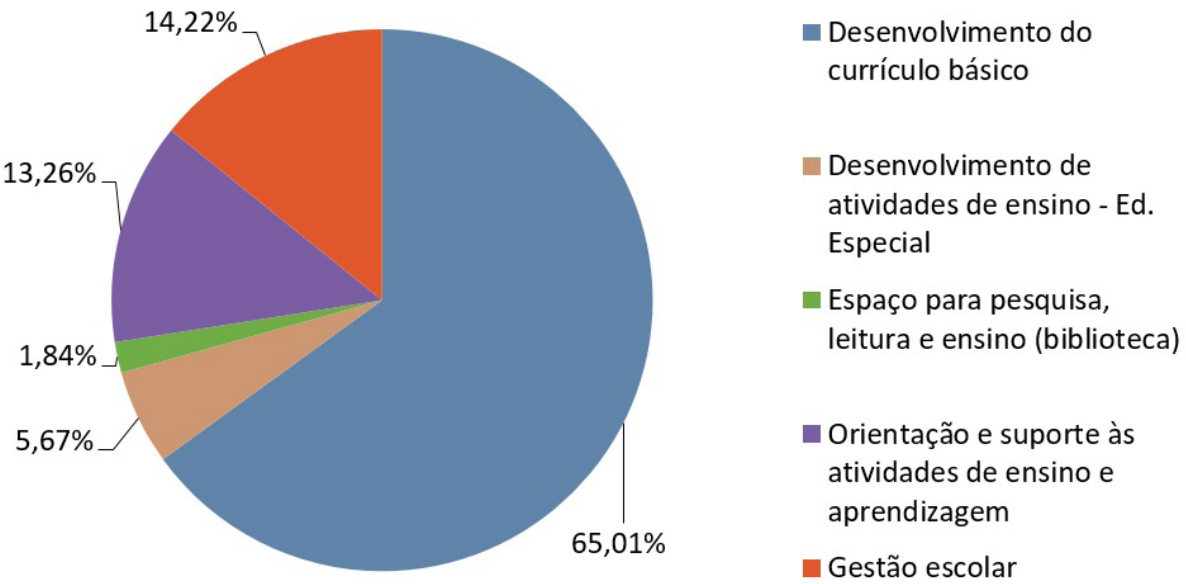

Gestão escolar

Fonte: Elaborado, pelo autor, com base em informações fornecidas pela Secretaria de Educação de SBC. 
Em relação à distribuição das notas em matemática na Prova Brasil de 2013, ainda que seja possível observar uma concentração das escolas entre 216 e 245 pontos na escala de matemática da Prova Brasil, pode-se notar certa dispersão das notas entre as escolas da rede (Gráfico 2). Além disso, verifica-se uma associação positiva entre o desempenho em matemática e o INSE das escolas, ou seja, quanto maior o nível socioeconômico médio dos alunos das escolas, maior tende a ser a pontuação observada na prova de matemática da Prova Brasil de 2013 (Gráfico 3).

Gráfico 2- Distribuição das escolas segundo a nota em matemática - rede municipal de SBC

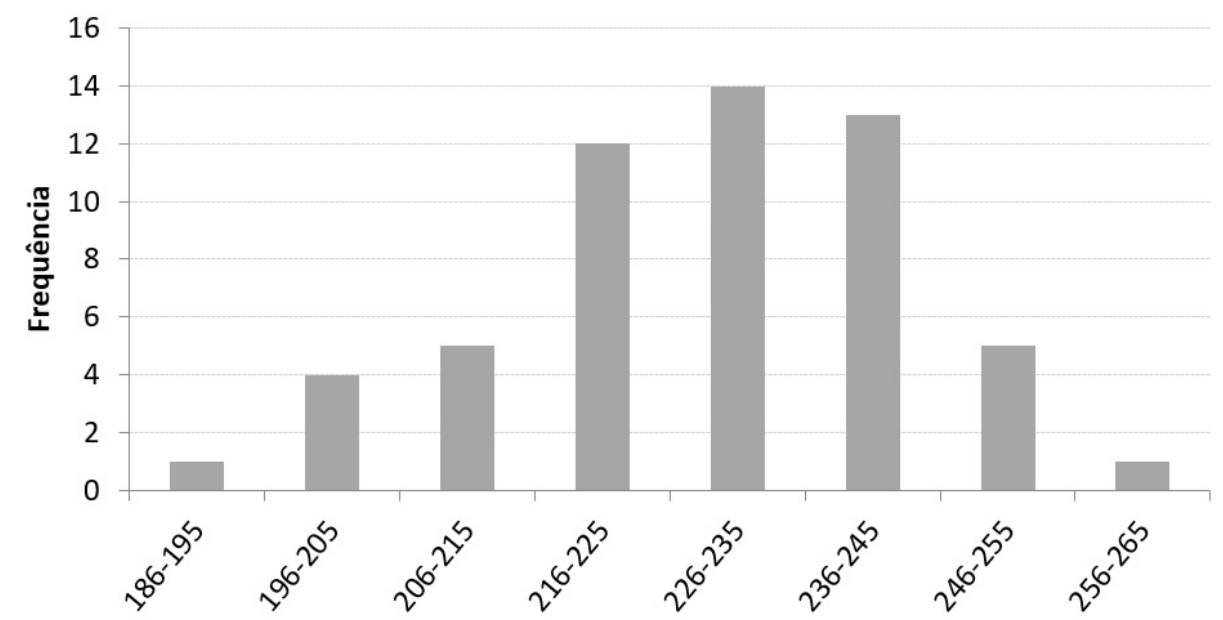

Faixas de desempenho em matemática - Prova Brasil

Fonte: Elaborado, pelo autor, com base nos dados da Prova Brasil de 2013.

Gráfico 3- Desempenho em matemática e nível socioeconômico - escolas da rede municipal de SBC

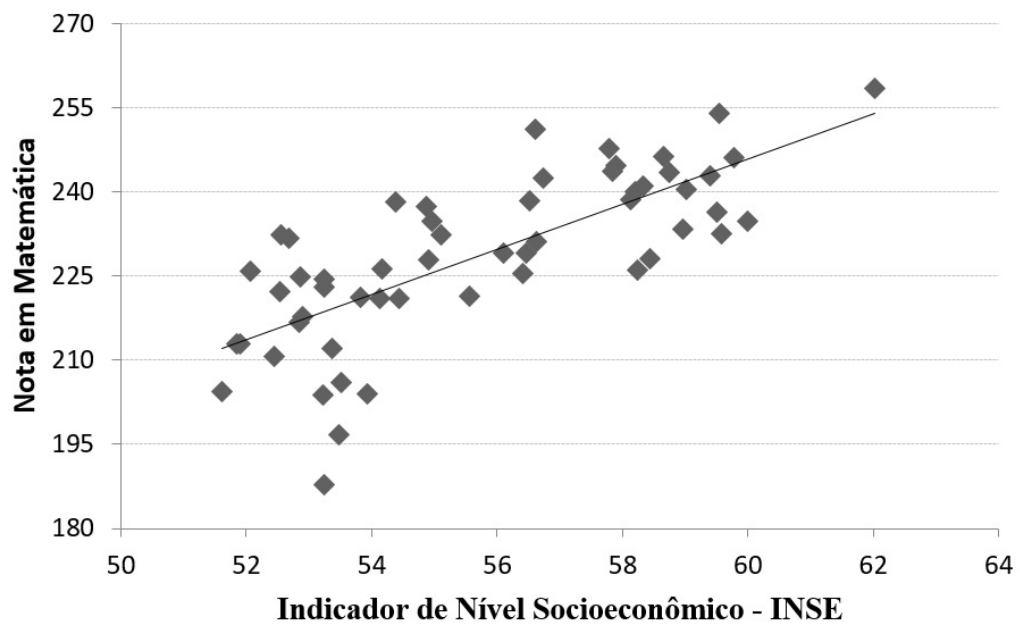

Fonte: Elaborado com base nas informações da Prova Brasil de 2013. 
Para a análise de como gastos e desempenho se relacionam, foram estimados seis modelos (Tabela 2). Nas duas primeiras colunas, que consideram o gasto total por aluno, é possivel notar a ausência de qualquer relação entre gastos e o desempenho dos alunos. Além disso, como era esperado, observa-se forte relação entre o nível socioeconômico e o desempenho. Caso a única informação disponível fosse o gasto total por aluno, a conclusão seria a de que o gasto não está associado ao desempenho, no contexto em questão. A comparação das duas primeiras colunas com as três restantes, que correspondem às melhores especificações obtidas, ilustra o potencial da informação de gasto desagregada, no nível da escola, para a compreensão das relações entre gastos e desempenho.

Tabela 2- Resultados das regressões - variável dependente: nota em matemática na prova Brasil de 2013

\begin{tabular}{|c|c|c|c|c|c|}
\hline & (1) & (2) & (3) & (4) & (5) \\
\hline Gasto total por aluno & $\begin{array}{l}-0.000 \\
(0.006)\end{array}$ & $\begin{array}{l}-0.005 \\
(0.004)\end{array}$ & & & \\
\hline \multirow[t]{2}{*}{ professores } & & & $-0.025^{* *}$ & -0.013 & -0.012 \\
\hline & & & $(0.011)$ & $(0.008)$ & $(0.008)$ \\
\hline \multirow[t]{2}{*}{ assist. classe } & & & $-0.094^{*}$ & $-0.057^{\star}$ & $-0.062^{*}$ \\
\hline & & & $(0.047)$ & $(0.033)$ & $(0.034)$ \\
\hline \multirow[t]{2}{*}{ coordenação } & & & $0.071^{*}$ & $0.050^{*}$ & $0.061^{+*}$ \\
\hline & & & $(0.036)$ & $(0.025)$ & $(0.025)$ \\
\hline \multirow[t]{2}{*}{ direção } & & & 0.018 & -0.010 & -0.020 \\
\hline & & & (0.019) & $(0.014)$ & $(0.014)$ \\
\hline \multirow[t]{2}{*}{ inse } & & $4.121^{1+*}$ & & $3.839^{*+*}$ & $3.361^{* *+}$ \\
\hline & & $(0.499)$ & & $(0.526)$ & $(0.527)$ \\
\hline \multirow[t]{2}{*}{ expdir 3 a 10} & & & & & $6.573^{\star}$ \\
\hline & & & & & (3.349) \\
\hline \multirow[t]{2}{*}{ expdir $11+$} & & & & & $9.083^{* *}$ \\
\hline & & & & & (3.762) \\
\hline \multirow[t]{2}{*}{ tempo disciplina } & & & & & 6.197 \\
\hline & & & & & $(4.929)$ \\
\hline \multirow[t]{2}{*}{ constante } & $229.618^{*+* *}$ & 9.526 & $247.739^{*+*}$ & 27.229 & 45.373 \\
\hline & (12.693) & (27.995) & $(14.897)$ & $(31.947)$ & (31.462) \\
\hline núm. & 55 & 55 & 55 & 55 & 54 \\
\hline R2 & 0.000 & 0.567 & 0.170 & 0.603 & 0.671 \\
\hline R2 ajus & -0.019 & 0.550 & 0.104 & 0.562 & 0.613 \\
\hline prob $>F$ & 0.942 & 0.000 & 0.049 & 0.000 & 0.000 \\
\hline
\end{tabular}

Fonte: elaboração do autor. Erro padrão em parênteses. Significância dos coeficientes: ${ }^{* \star \star} 1 \%$; ${ }^{* \star} 5 \%$; ${ }^{*} 10 \%$. Nota: As variáveis "expdir 3 a 10 " e "expdir $11+$ " indicam que a escola conta com diretores com 3 a 10 anos de experiência nesta função ou com 11 anos ou mais de experiência nessa função, respectivamente. 
Considerando que as especificações melhoraram na medida em que os gastos foram desagregados, os modelos finais (colunas 3 a 5$)^{13}$ utilizaram dados de gasto por aluno segundo as categorias de menor agregação, conforme propostas. Em relação a estas, a opção foi excluir Suporte aos alunos e professores e Apoio operacional e administrativo, pressupondo que tendem a ter menos relevância que as demais para a explicação do desempenho dos alunos. A falta de significância estatística dessas variáveis e a melhoria da especificação dos modelos após sua exclusão validaram, de certa forma, a opção feita. Também foram excluídos da análise os gastos relativos à Educação especial, pois estes gastos estão essencialmente relacionados à necessidade de recursos de cada escola, não havendo motivos para esperar uma associação entre recursos e desempenho.

Os resultados dispostos nas colunas 4 e 5, que incluem o controle do nível socioeconômico dos alunos, indicam associação positiva e estatisticamente significante entre gastos com Coordenação pedagógica e o desempenho dos alunos em matemática e negativa e significante entre gastos com Assistentes de classe ${ }^{14}$ e desempenho.

Tendo em vista o coeficiente obtido para Coordenação pedagógica, pode-se dizer que uma variação de, por exemplo, $\mathrm{R} \$ 10,00$ - que não é alta, considerando um gasto médio de $\mathrm{R}$ \$ 203,44 nesta categoria - está associada a um acréscimo de 0,5 a 0,61 na nota em matemática das escolas da rede considerada. Em relação ao gasto com Assistentes de classe, a mesma variação de $R$ \$ 10,00 no gasto por aluno está associada a uma variação negativa de aproximadamente 0,6 na nota média em matemática. 0 resultado obtido não permite, contudo, recomendações acerca de como a rede considerada deve alocar seus recursos.

Em primeiro lugar, a abordagem utilizada não permite a inferência de causalidade. Isto é, não se pode dizer, por exemplo, que um aumento do gasto com Assistentes de classe impactaria negativamente o desempenho dos alunos. Para este tipo de análise, seria necessária outra abordagem metodológica, consideradas determinadas condições ${ }^{15}$. Em segundo lugar, a relação negativa observada não significa que os gastos com Assistentes de classe não são relevantes. Eles tanto podem estar positivamente associados a algum tipo de resultado escolar não levado em conta, quanto podem viabilizar atividades escolares essenciais para o desenvolvimento de atividades escolares que não estejam associadas a qualquer tipo de resultado, mas que implicam alguma forma de realização do direito à educação. Nesse último caso, esses gastos representariam um bem em si, independente de qualquer resultado que se espere da educação escolar.

Independente das conclusões que os coeficientes estimados permitem ou não chegar, pode-se destacar que a relação entre gastos e desempenho não tem um sentido único, como frequentemente se conclui. A desagregação do gasto por aluno segundo as categorias propostas indica que a constatação de que gastos estão ou não associados ao desempenho

13- Os testes de Breusch-Pagan e Shapiro-Wilk confirmaram as hipóteses de homocedasticidade e de normalidade dos resíduos, respectivamente, para as três últimas especificações da Tabela 2. Os resultados obtidos para o Fator de Inflação da Variância - VIF indicaram não haver problemas de multicolinearidade.

14- Considerando a função exercida e o pouco número de ocorrências, os gastos com estagiários (que correspondem a menos de $0,3 \%$ dos gastos da categoria Desenvolvimento do currículo básico) foram incorporados à categoria Assistentes de classe.

15 - Para a inferência de causalidade, a utilização de variáveis instrumentais seria uma abordagem possível. Por exemplo, a ocorrência de uma situação em que houvesse alguma medida de política educacional do município que implicasse variação do gasto e que, simultaneamente, não estivesse associada a alguma variável não observada, poderia viabilizar este tipo de abordagem. 
dos alunos pode ser restrita. Isto é, os diferentes sentidos, grandezas e significâncias dos coeficientes estimados parecem apontar que diferentes tipos de gastos, associados a diferentes atividades escolares, estão relacionados de diferentes formas ao desempenho. E (re)conhecer estas diferenças pode ser um passo importante para o entendimento de como os recursos destinados à educação podem contribuir para a melhoria do ensino.

\section{Considerações finais}

Este artigo teve como propósito explorar a relação entre gastos com educação e desempenho, tendo como base a informação de gasto por aluno no nível da escola e segundo categorias de gastos que indiquem não os insumos utilizados pelas escolas, mas as atividades ou funções desempenhadas por elas. Para isso, foram propostas categorias de gasto para as despesas com o pagamento de salários dos profissionais que atuam nas escolas da rede municipal de ensino de São Bernardo do Campo e, em seguida, foram exploradas as relações entre o gasto por aluno e o desempenho das escolas em matemática na Prova Brasil de 2013, controlado o nível socioeconômico dos alunos.

Os resultados indicaram que diferentes tipos de gasto estão associados de diferentes formas ao desempenho e que os modelos estimados melhoram na medida em que os gastos são desagregados. Em relação aos coeficientes estimados, observou-se relação positiva entre gastos por aluno com coordenação escolar e a nota em matemática e relação negativa entre os gastos por aluno com assistentes de classe e o desempenho na mesma matéria. Além disso, como era esperado, o nível socioeconômico dos alunos se mostrou relevante para explicar o desempenho dos alunos.

Não entendemos que as relações observadas, contudo, permitam que sejam tecidas recomendações sobre como a rede municipal de São Bernardo do Campo deve alocar seus recursos, já que se fossem considerados outros tipos de resultados - que não o desempenho em testes de larga escala - as relações observadas poderiam ser outras. Além disso, tendo em vista a perspectiva da educação como um direito, pode-se entender que os gastos necessários para a viabilização do ensino devem ser sempre garantidos, independente do que se possa esperar em termos de resultados.

Além do exposto, vale indicar que as estimativas foram feitas com base em um número restrito de observações, ainda que este número (55) corresponda à boa parte do universo considerado de escolas (61 escolas que ofereciam exclusivamente o ensino fundamental). Desta forma, há necessidade da realização de outros trabalhos, com base em maior número de dados, que possam utilizar maior número de variáveis de controle e que avancem em abordagens que permitam a inferência de causalidade.

É importante também sinalizar que pesquisas qualitativas são essenciais para o entendimento de como as redes de ensino decidem sobre a alocação de seus recursos e de como as escolas efetivamente utilizam os recursos a elas destinados. Isto é, são necessárias abordagens que explorem como as escolas usam os recursos disponíveis, como computadores, laboratórios, bibliotecas e quadras, ou como o tempo na escola se distribui, por exemplo, entre aulas que abrangem o currículo básico, reforço escolar, reuniões pedagógicas etc. 
Por fim, destacamos que, para além do papel dos recursos escolares no desempenho dos alunos, são necessárias análises que revelem em que medida as escolas e redes de ensino têm viabilizado o atendimento das necessidades educativas dos alunos, distribuindo recursos segundo as necessidades de aprendizagem de cada um, considerando as desigualdades com que os alunos chegam às escolas. Pesquisas que explorem, no interior de uma mesma rede de ensino e entre alunos de uma mesma escola, aspectos relativos à equidade na distribuição dos recursos, tendo em vista a condição social dos alunos, podem contribuir para a construção de caminhos que levem à realização do direito à educação de qualidade para todos.

\section{Referências}

ALVES, Maria Teresa Gonzaga; FRANCO, Creso. A pesquisa em eficácia escolar no Brasil: evidências sobre 0 efeito das escolas e fatores associados à eficácia escolar. In: BROOKE, Nigel; SOARES, José Francisco (Org.). Pesquisa em eficácia escolar: origem e trajetórias. Belo Horizonte: UFMG, 2008. p. 482-500.

ALVES, Thiago. Desenvolvimento de um modelo de previsão de custos para planejamento de sistemas públicos de educação básica em condições de qualidade: uma aplicação a municípios de Goiás. 2012. Tese (Doutorado) - Faculdade de Economia, Administração e Contabilidade da Universidade de São Paulo, São Paulo, 2012.

AMARAL, Luiz Felipe Leite Estanislau do Amaral. Os determinantes dos gastos educacionais e seus impactos sobre a qualidade do ensino. 2011. Dissertação (Mestrado) - Faculdade de Economia, Administração e Contabilidade da Universidade de São Paulo, São Paulo, 2011.

AMARAL, Luiz Felipe Leite Estanislau do Amaral; MENEZES-FILHO, Naércio. A relação entre gastos educacionais e desempenho escolar. In: ENCONTRO NACIONAL DE ECONOMIA, 36., 2008, Salvador. Anais eletrônicos... Salvador: ANPEC, 2008. Disponível em: <http://www.anpec.org.br/encontro2008/ artigos/200807201800160-.pdf>. Acesso em: 18 jun. 2017. p. 0-19.

ARCHIBALD, Sarah. Narrowing in on educational resources that do affect student achievement. Peabody Journal of Education, Philadelphia, v. 81, n. 4, p. 23-42, out. 2006.

BAUER, Adriana; ALAVARSE, Ocimar Munhoz; OLIVEIRA, Romualdo Portela. Avaliações em larga escala: uma sistematização do debate. Educação e Pesquisa, São Paulo, v. 41, n. especial, p. 1367-1382, dez. 2015.

BENEVIDES, Maria Victoria de Mesquita. Educação para a democracia. Lua Nova, São Paulo, n. 38, p. 223-237, dez. 1996.

BONAMINO, Alicia; SOUSA, Sandra Zákia. Três gerações de avaliação da educação básica no Brasil: interfaces com 0 currículo da/na escola. Educação e Pesquisa, São Paulo, v. 38, n. 2, p. 373-388, abr./jun. 2012. 
CARVALHO, Malena Xavier. Processos escolares como indicadores de qualidade em educação: um estudo a partir da formação para cidadania. 2015. Dissertação (Mestrado em Educação) - Faculdade de Educação da Universidade de São Paulo, São Paulo, 2015.

CHUDGAR, Amita; LUSCHEl, Thomas F. National income, income inequality, and the importance of schools: a hierarchical cross-national comparison. American Educational Research Journal, v. 46, n. 3, p. 626-658, set. 2009.

COLEMAN, James S. et al. Equality of educational opportunity. Washington, DC: National Center for Educational Statistics, 1966.

CONLEY, David T.; PICUS, Lawrence 0. Oregon's quality education model: linking adequacy and outcomes. Educational Policy, v. 17, n. 5, p. 586-612, nov. 2003.

FERGUSON, Ronald F. Paying for public education: new evidence on how and why money matters. Harvard Journal on Legislation, v. 28, p. 465-498, 1991.

GALVÃO, Fernando Vizotto. Gastos com educação e desempenho escolar: uma análise no nível da escola. 2016. Dissertação (Mestrado em Educação) - Faculdade de Educação da Universidade de São Paulo, São Paulo, 2016.

HAIR, Joseph F. et al. Análise multivariada de dados. Porto Alegre: Artmed, 2009.

HANUSHEK, Eric A. Assessing the effects of school resources on student performance: an update. Educational Evaluation and Policy Analysis, v. 19, n. 2, p. 141-164, 1997.

HANUSHEK, Eric A. The economics of schooling: production and efficiency in public schools. Journal of Economic Literature, v. 24, n. 13, p. 1141-1177, 1986.

HANUSHEK, Eric A. The failure of input-based schooling policies. The Economic Journal, v. 113, n. 485, p. 64-98, fev. 2003.

HANUSHEK, Eric A. The impact of differential expenditures on school performance. Educational Researcher, v. 18, n. 4, p. 45-51, maio. 1989.

HANUSHEK, Eric A.; RIVKIN, Steven G.; TAYLOR, Lori L. Aggregation and the estimated effects of school resources. The Review of Economics and Statistics, v. 78, n. 4, p. 611-627, nov. 1996.

HEDGES, Larry; LAINE, Richard D.; GREENWALD, Rob. Does money matter? A meta-analysis of the effects of differential schools inputs on student outcomes. Educational Researcher, v. 23, n. 3, p. 5-14, abr. 1994.

INEP. Instituto Nacional de Estudos e Pesquisas Educacionais Anísio Teixeira. Indicador de Nível Socioeconômico das Escolas (INSE): nota técnica. Brasília, DF: INEP, 2014. Disponível em: <http://download. inep.gov.br/informacoes_estatisticas/indicadores_educacionais/2011_2013/nivel_socioeconomico/ nota_tecnica_indicador_nivel_socioeconomico.pdf>. Acesso em: 25 jun. 2017. 
KIMBALL, Rhonda L. Where does the money go? An analysis of student level resource allocation at the school level. 2009. (PhD in Education) - School of Education, University of Southern California, California, 2009.

LEVY, Samuel; CAMPINO, Antonio Carlos; NUNES, Egas Moniz. Análise econômica do sistema educacional de São Paulo. São Paulo: IPE-USP, 1970.

MONTEIRO, Joana. Gasto público em educação e desempenho escolar. Revista Brasileira de Economia, Rio de Janeiro, v. 69, n. 4, p. 467-488, out./dez. 2015.

ODDEN, Allan et al. Defining school-level expenditure structures that reflect educational strategies. Journal of Education Finance, v.28, n.3, p. 323-356, 2003.

OLIVEIRA, Romualdo Luiz Portela de. A pesquisa em financiamento da educação. In: GOUVEIA, Andrea Barbosa; SOUZA, Angelo Ricardo; TAVARES, Thaís Moura (Org.). Conversas sobre financiamento da educação. Curitiba: UFPR, 2006. p. 13-26.

OLIVEIRA, Romualdo Luiz Portela de; ARAUJO, Gilda Cardoso de. Qualidade do ensino: uma nova dimensão da luta pelo direito à educação. Revista Brasileira de Educação, Rio de Janeiro, n. 28, p. 5-23, jan./abr. 2005.

OLIVEIRA, Romualdo Luiz Portela de et al. Análise das desigualdades intraescolares no Brasil. Estudos \& pesquisas educacionais, São Paulo: Fundação Victor Civita, 2013. p. 19-112.

PAR0, Vitor Henrique. Estudo comparativo de custo-aluno nos diversos graus e modalidades de ensino. São Paulo: ATPCE/FCC, 1981.

PICUS, Lawrence 0. Does money matter in education? A policymaker's guide. Selected Papers in School Finance, Washington, DC: National Center for Education Statistics, p. 16-36, 1995.

PICUS, Lawrence 0; PETERNICK, Lauri. Developing student resource variables for the early childhood longitudinal survey. Developments in School Finance, Washington, DC: National Center for Education Statistcs, p. 107-128, 1998.

PICUS, Lawrence et al. Implementing school finance adequacy: school level resource in Wyoming following adequacy-oriented finance reform. California: Lawrence 0. Picus and Associates, 2008.

VERHINE, Robert E. Custo-aluno-qualidade em escolas de educação básica: 2ª etapa. Brasília, DF: INEP, 2006.

VERHINE, Robert E. Determinação de custos educacionais: uma análise panorâmica do estado da arte. Revista Educação, Porto Alegre, v. 21, n. 35, p. 107-122, 1998.

VIGNOLES, Anna et al. The relationship between resource allocation and pupil attainment: a review. London: Centre for Economics of education, 2000.

XAVIER, Antônio Carlos; MARQUES, Antônio Emilio S. Custo direto de funcionamento das escolas públicas de $1^{\circ}$ grau da região sul. Brasília, DF: MEC/SEB, 1988. 
Recebido em: 25.09.2017

Revisões em: 13.11.2017

Aprovado em: 15.12.2017

Fernando Vizotto Galvão é mestre em Educação pela Universidade de São Paulo, graduado em Ciências Econômicas pela Universidade de São Paulo e atua como Especialista em Políticas Públicas no Governo do Estado de São Paulo.

\section{Anexos}

Anexo A- Tabela de indicadores de recursos, conforme proposta por Odden et al. (2003)

\begin{tabular}{|c|c|}
\hline \multicolumn{2}{|l|}{ School Resource Indicators } \\
\hline School Building Size & Length of Reading Class (Elementary) \\
\hline School Unit Size & Length of Mathematics Class (Elementary) \\
\hline Percent Low Income & Reading Class Size (Elementary) \\
\hline Percent Special Education & Mathematics Class Size (Elementary) \\
\hline Percent ESL/LEP & Regular Class Size (Elementary) \\
\hline Expenditures Per Pupil & Length of Core* Class Periods (Secondary) \\
\hline Professional Development (expenditures per teacher) & Core Class Size (Secondary) \\
\hline Special Academic Focus of School/Unit & Non-Core Class Size (Secondary) \\
\hline Length of Instructional Day & Percent Core Teachers \\
\hline \multicolumn{2}{|l|}{ Length of Class Periods } \\
\hline & ${ }^{*}$ Math, English/LA, Science, \& Social Studies \\
\hline
\end{tabular}

Fonte: ODDEN et al., 2003, p. 330. 
Fernando Vizotto GALVÃO

Anexo B- Tabela de estrutura de gastos escolares, conforme proposta por Odden et al. (2003).

Instructional

1. Core Academic

Teachers English/ Reading/ Language Arts

History/ Social Studies

Math

Science

2. Specialist and Elective Teachers/Planning and Preparation

Art, music, physical education, etc.

Academic Focus with or without Special Funding

Vocational

Drivers Education

Librarians

\section{Extra Help}

Tutors

Extra Help Laboratories

Resource Rooms (Title I, special education or other part-day pull- out programs)

Inclusion Teachers

English as a second language classes

Special Education self-contained classes for severely disabled students (Including aides)

Extended Day and Summer School

District-Initiated Alternative Programs

4. Professional Development

Teacher Time - Substitutes and Stipends

Trainers and Coaches

Administration

Materials, Equipment and Facilities

Travel \& Transportation

Tuition and Conference Fees

5. Other Non-Classroom Instructional Staff

Coordinators and Teachers on Special Assignment

Building Substitutes and Other Substitutes

Instructional Aides

6. Instructional Materials and Equipment

Supplies, Materials and Equipment

Computers (hardware, software, peripherals)

\section{Student Support}

Counselors

Nurses

Psychologists

Social Workers

Extra-Curricular and Athletics

Non-Instructional

\section{Administration}

9. Operations and Maintenance

Custodial

Utilities

Security

Food Service

Fonte: ODDEN et al., 2003, p. 330. 
Anexo C- categorias de gasto e funções escolares - rede municipal de SBC.

\begin{tabular}{|c|c|c|}
\hline Categoria & Subcategoria & Função \\
\hline \multirow{6}{*}{$\begin{array}{l}\text { Desenvolvimento do } \\
\text { currículo básico }\end{array}$} & \multirow{4}{*}{ Professores } & Professor de Educação Básica - Fundamental \\
\hline & & Professor de Educação Básica I - Fundamental \\
\hline & & Professor de Educação Básica - Infantil \\
\hline & & Professor de Educação Básica I - Infantil \\
\hline & Assistentes de classe & Auxiliar em Educação I \\
\hline & Estagiários & Estagiário em Pedagogia \\
\hline \multirow{3}{*}{$\begin{array}{l}\text { Desenvolvimento de } \\
\text { atividades de ensino - } \\
\text { Educação Especial }\end{array}$} & Professores Ed. Esp. & Professor de Educação Especial \\
\hline & \multirow{2}{*}{ Estagiários Ed. Esp. } & Estagiário de Apoio à Inclusão (Pedagogia) \\
\hline & & Estagiário de Apoio à Inclusão \\
\hline \multirow{5}{*}{$\begin{array}{l}\text { Espaço para pesquisa, } \\
\text { leitura e ensino }\end{array}$} & \multirow{5}{*}{$\begin{array}{c}\text { Espaço para pesquisa, leitura } \\
\text { e ensino }\end{array}$} & Agente de Biblioteca e Arquivo \\
\hline & & Auxiliar Administrativo de Ensino - Biblioteca Escolar Interativa \\
\hline & & Auxiliar de Biblioteca \\
\hline & & Oficial Administrativo - Biblioteca Escolar Interativa \\
\hline & & Oficial de escola - Biblioteca Escolar Interativa \\
\hline \multirow{6}{*}{$\begin{array}{l}\text { Orientação e suporte às } \\
\text { atividades de ensino e } \\
\text { aprendizagem }\end{array}$} & \multirow{4}{*}{ Coordenação Pedagógica } & Coordenador Pedagógico \\
\hline & & Professor Coordenador Conveniado \\
\hline & & Professor Respondendo pela Coordenação Pedagógica \\
\hline & & Professor de Apoio aos Programas Educacionais \\
\hline & \multirow{2}{*}{ Suporte aos alunos e professores } & Inspetor de Alunos \\
\hline & & Monitor em educação \\
\hline \multirow{11}{*}{ Gestão escolar } & \multirow{6}{*}{ Direção da escola } & Diretor Escolar \\
\hline & & Diretor Escolar Conveniado \\
\hline & & Professor de Apoio à Direção Escolar \\
\hline & & Professor Respondendo por Direção Escolar \\
\hline & & Vice-diretor Conveniado \\
\hline & & Readaptado \\
\hline & \multirow{5}{*}{$\begin{array}{l}\text { Apoio operacional e } \\
\text { administrativo }\end{array}$} & Ajudante Geral \\
\hline & & Merendeira \\
\hline & & Oficial Administrativo \\
\hline & & Oficial de Escola \\
\hline & & Servente \\
\hline
\end{tabular}

Fonte: Elaborado com base em informações fornecidas pela Secretaria de Educação de SBC. 